\title{
Mutual Informative Brown Clustering based Multiattribute Cockroach Swarm Optimization for Reliable Data Dissemination in VANET
}

\author{
Mrs D.Radhika ${ }^{1}$ \\ Research Scholar \\ Cauvery College for Women (Autonomous) \\ Affiliated to Bharathidasan University \\ Tiruchirappalli, Tamilnadu \\ India
}

\author{
Dr. A.Bhuvaneswari ${ }^{2}$ \\ Associate Professor \\ Cauvery College for Women (Autonomous) \\ Affiliated to Bharathidasan University \\ Tiruchirappalli, Tamilnadu \\ India
}

\begin{abstract}
A vehicular ad hoc network (VANETs) intends to obtain communication for vehicular networks and enhances road safety and effectiveness with help of wireless technology. Data dissemination is an important process in communication. In VANETs system, Data dissemination plays a significant role. A novel Mutual informative brown clustering-based multi attribute cockroach swarm optimization (MIBCMCSO) technique is introduced for improving data dissemination. In reliable data dissemination, clustering and optimization are the two major process of proposed MIBCMCSO technique. Initially, clustering procedure is performed for separating entire network towards different groups of vehicle nodes namely distance, direction, density and velocity of node. For each group, cluster head was chosen among the members to efficient data with minimum delay. Secondly, multi attribute cockroach swarm optimization technique is applied for finding optimal cluster head through multi attribute functions such as residual energy, bandwidth availability, and distance. Then source node performs data dissemination destination via optimal cluster head. Simulation of MIBCMCSO as well as existing technique is performed by various performance parameters like packet delivery ratio, end to end delay and throughput. MIBCMCSO achieves higher consistency of data dissemination as well as lesser delay than conventional methods.
\end{abstract}

Keywords-VANET; data dissemination; mutual informated brown agglomerative clustering; multi attribute cockroach swarm optimization

\section{INTRODUCTION}

VANET is a wireless communication system used for disseminating multimedia information from one vehicle to other vehicles in the dynamic structure of the network. Reliable data dissemination is a significant process in a wireless network. To address this issue, a cluster-based optimization technique is introduced. Fig. 1 specifies the various types of routing protocols in VANET.

A new cluster-based reliable routing scheme called CEGRAOD was developed by Zahid Khan et al., (2019) [1] to find the most reliable path from the source to destination. A criterion for cluster members (CMs) and cluster heads $(\mathrm{CHs})$ selection. The CVoEG model divides VANET nodes (vehicles) into an optimal number of clusters (ONC) used by
Eigen gap heuristic. The cluster includes vehicle was selected as a $\mathrm{CH}$ for maximum Eigen-centrality score. But, the method failed to use optimization technique for select the optimal cluster head. A multi valued DPSO is designed in [2] for identifying data dissemination from source to destination vehicle. In order to develop detection of optimal path in VANETs, Multi valued Discrete Particle Swarm Optimization (DPSO) was employed. While considering the more packets for dissemination, the performance of delay is not reduced.

Clustering and Probabilistic Broadcasting (CPB) method is developed in [3] for data dissemination. A clustering algorithm was to constrain the directions of vehicles exchange their data in a clustered way with adequate association period. In the created clustering structure was to broadcast the data between vehicles. Each cluster associate forwards the received packet to its cluster head was associated with the number of times the same packet was received through one interval. But, this approach minimizes delay, throughput is not improved. Novel data dissemination for VANETs was introduced in [4] for disseminating emergency messages with different traffic scenarios. In order to choose next forwarding vehicle (NFV), the segmentation of vehicle uses DDP4V. Though the efficient and reliable data dissemination protocol is designed for controlling data dissemination in highway and urban VANET scenarios, designed protocol failed to perform reliable data dissemination.



Fig. 1. Type of VANET Routing Protocol. 
Data Dissemination protocol is introduced in [5] for data dissemination. Path-based clustering data dissemination protocol (PCDP) consists of two parts. The first part was a clustering formation clusters based on the expected path of the vehicle. The survivability of the cluster was enhanced by created cluster among nodes with the minimum difference by evaluated the designed paths of the vehicles. But, protocol reduces delay while using higher vehicle density, the reliability of data transmission remained unaddressed. An intelligent clustering-based optimization method was introduced in [6] to enhance the reliable data transmission for each vehicle. The moth flame optimization framework was optimizing the cluster and primary focus of the scheme was to improve the power in vehicular ad hoc networks. Flame optimization was proved by two variants of particle swarm optimization such as multiple-objective particle swarm optimization and comprehensive learning particle swarm optimization and a variant of ant colony optimization. But the designed optimization method failed to consider the multiobjective functions for solving the data dissemination.

Analytical network process (ANP) is introduced in [7] using the multi criteria tool for data dissemination through the optimal vehicle nodes. The various performance metrics such as reliability, delay remained unsolved. The stability of different candidate vehicles for NFV (Next Forwarder Vehicle) selection level was verified for sensitivity analysis of dissimilar development But, ANP method minimizes the latency but the reliable data dissemination was not performed. Passive data dissemination method is designed in [8] using cluster-based method. However, the energy-aware reliable data dissemination remained unsolved in VANET. The approach was consist on each vehicle for transmit periodical measures about the position, speed to other vehicles were the same signal range and same cluster. The previous division of the network into virtual sub-groups was easy management and data dissemination of messages. A replication-based distributed randomized approach was introduced in [9] to disseminate the information. Amount of data extend in network is restricted for decreasing transmission as well as increasing data dissemination delay. The approach failed to use cluster-based data dissemination in order to further minimize the delay. A hybrid method was developed in [10] for resolving utility-based maximization and choose data dissemination. The utility function was taken by the delivery delay, Quality of Service (QoS) and storage cost. With precise analysis was obtained the closed-form of the expected utility of a path and then attain the optimal solution of the problem with the curved optimization theory. But the method failed to achieve higher reliability in the data distribution. An Adaptive Data Dissemination Protocol (ADDP) was introduced in [11] for providing reliability to message transmission. In order to minimize communication and beacons in network, designed method employs various method for vigorously regulate beacon periodicity. Though the vehicles faces failures in message delivery, the vehicle movement direction was not considered.

Hybrid relay node selection method is presented in [12] to multi hop data dissemination with minimum delay as well as bandwidth utilization. The new hybrid scheme was obtain the spatial allocation of the next-hop transmitted nodes with location to the present sending node. A hybrid scheme was that attempts to improve performance of VANETs over unstable node densities, traffic load conditions and mobility speed scenarios. While the chosen relay nodes is controlled for exploiting single selection criterion, the energy-based relay nodes selection is not carried out. Named Data Networking (NDN) approach is designed in [13] to deliver message contents with higher network throughput. A new protocol named Roadside Unit (RSU) assisted of Named Data Network (RA-NDN) was operating as a standalone node standalone RSU (SA-RSU)]. The approach was reduces the different vehicular densities, vehicular communication ranges and number of requesters with vehicular NDN via a real-world data set. But the performance of delay was not effectively reduced since it failed to use the clustering technique. Integrated message dissemination and traffic regulation were performed in [14] with higher network throughput. On-ramp traffic flow control method is used for controlling vehicles towards highway. But the packet delivery ratio was not improved. On-Demand Member-Centric Routing (OMR) protocol was developed in [15] for data dissemination. Routing protocols is employed for video streaming service at certain platoon member. However, the performance of the delay was not reduced.

A hybrid-fuzzy logic guided genetic algorithm (H-FLGA) approach was proposed in [16] for the software defined networking controller, to solve a multi-objective resource optimization problem for 5G driven VANETs. A Multi access Edge Computing (MEC) based delay-constrained k-hoplimited VANET data offloading method was developed in [17] to derive the potential V2V-V2I paths. TBD (Trajectory Based Dissemination) solution that transmits the information on the network according was proposed in [18] to the density of vehicles in the path from the source to the region of interest. A Multi-hop Cooperative Data Dissemination (MHCDD) based on buffer control was introduced in [19] which can be more efficient when applied with a Markov process. An ad-hocbased solution was designed in [20] for V2V charging where both information dissemination and charging pairs allocation are performed over VANET. A hybrid data dissemination model with both vehicle-to-vehicle (V2V) and vehicle-toinfrastructure (V2I) disseminations was proposed in [21] to reduce the traffic on the edge, in which the edge (infrastructure) selectively injects data to the vehicles and leverages the vehicle network to disseminate the data.

\section{A. Major Contribution}

The major contribution of the work MIBCMCSO technique is summarized as follows:

To improve the reliability of data dissemination in VANET, the MIBCMCSO technique is introduced. This contribution is achieved by performing the cluster based optimal cluster head identification. The mutual informated brown agglomerate clustering is applied to group the vehicle nodes based on the moving direction, velocity, and distance.

To improve the throughput, the multi attribute cockroach swarm optimization technique is applied for finding the optimal cluster head between source and destination based on 
energy, distance, and bandwidth availability. The data dissemination is carried out via an optimal cluster head. This assists to minimize end to end delay of data dissemination.

\section{B. Outline of Paper}

The rest of paper is organized into five different sections. Section 2 describes the proposed methodology MIBCMCSO with a neat diagram. In Section 3, simulation settings are presented and various results of the different parameters are discussed in Section 4. Finally, the conclusion of the paper is presented in Section 5.

\section{METHODOLOGY}

An efficient algorithm called MIBCMCSO is introduced with the objective of improving data dissemination and minimizing the delay in VANET. The MIBCMCSO technique comprises the ' $n$ ' number of vehicle nodes to perform the data transmission within the communication range. The vehicle nodes are partitioned into ' $j$ ' number of clusters $c_{1}, c_{2}, c_{3} \ldots c_{j}$ and the cluster head $\left(\mathrm{N}_{\mathrm{H}}\right)$ is selected for disseminating the data packets from source vehicle $\left(S_{\mathrm{V}}\right)$ to destination vehicle $\left(D_{\mathrm{V}}\right)$. The flow process of the MIBCMCSO technique is shown in Fig. 1.

Fig. 2 illustrates the flow process of the proposed MIBCMCSO technique to obtain the better reliability of data dissemination with minimum delay. Initially, the clustering technique is applied for dividing the total network into different groups of vehicle nodes in the network based on the different characteristics such as vehicle density, direction, distance, and velocity. Then the data packet transmission is performed by selecting the cluster head using a multi attribute optimization technique. These two processes are clearly explained in the following sections.

A. Mutual informated brown agglomerative clustering. The first process of the proposed MIBCMCSO technique is to divide the total mobile nodes in the VANET into a number of groups. The mutual informated brown agglomerative clustering is the hierarchical clustering algorithm used to combine the two clusters into one move up the hierarchy. This clustering process is carried out based on their characteristics such as vehicle density, direction, distances, and velocities.

Initially, the vehicle moving directions from one location to another is computed. In the two dimensional space, the current coordinate of the vehicle is $\left(\mathrm{P}_{1}, \mathrm{P}_{2}\right)$ and previous coordinate of the same vehicle is $\left(\mathrm{Q}_{1}, \mathrm{Q}_{2}\right)$.

$\tan \theta=\left[\left(\mathrm{Q}_{2}-\mathrm{Q}_{1}\right) /\left(\mathrm{P}_{2}-\mathrm{P}_{1}\right)\right]$

where, ' $\tan \theta$ ' denotes a tangent function used to identify the moving direction of the node. Afterward, the distance between the two-vehicle nodes is measured using the given mathematical formula. The coordinates of one vehicle node are $\left(\mathrm{u}_{1}, \mathrm{v}_{1}\right)$ and coordinates of another vehicle node $(\mathrm{u} 2, \mathrm{v} 2)$.

$\alpha\left(\tau_{\mathrm{i}}, \tau_{\mathrm{j}}\right)=\sqrt{ }\left(\mathrm{u}_{2}-\mathrm{u}_{1}\right) 2+\left(\mathrm{v}_{2}-\mathrm{v}_{1}\right) 2$

Where, $\alpha\left(\tau_{\mathrm{i}}, \tau_{\mathrm{j}}\right)$ represent the distance between two vehicle nodes in the two-dimensional space in the network. Then the velocity of the vehicle node is calculated based on their movement in the given period of time. The mathematical for calculating the node velocity is given below:

$\tau_{\mathrm{vel}}=\left(\alpha_{\tau} / \mathrm{t}\right)$

Where, ' $\tau_{\mathrm{vel}}$ ' represents a velocity of the vehicle node, ' $\alpha_{\tau}$ ' denotes a distance moved by the node in a given time period (t). The node velocity is measured in meter per second $(\mathrm{m} / \mathrm{sec})$. Based on the above-said characteristics, Mutual informated brown agglomerative clustering is applied to group the vehicle nodes. The number of vehicle nodes ' $\tau_{1}, \tau_{2}, \tau_{3} \ldots \tau_{\mathrm{m}}$ ' are distributed in a transmission range. Initially, the proposed clustering algorithm initializes the ' $\mathrm{k}$ ' number of clusters in the given dimensional space. For each cluster, the mean (i.e. cluster centroid) is assigned to partitions the vehicle nodes.

The distance between the mean and the vehicle nodes is calculated. The Manhattan distance is calculated as given below. $\mathrm{k} \mathrm{n}$.

$\mathrm{d}_{\mathrm{ij}}=\sum \sum\left|\mathrm{c}_{\mathrm{j}}-\tau_{\mathrm{i}}\right|$

$\mathrm{j}=1 \mathrm{i}=1$

Where, $d_{i j}$ denotes a distance between the cluster mean $c_{j}$ and $\tau_{\mathrm{i}}$ represents the vehicle node. Then the node which is closer to the cluster mean is grouped into that particular cluster.

$\mathrm{Y}=\arg \min \mathrm{d}_{\mathrm{ij}}$

where $\mathrm{Y}$ denotes a clustering output, arg min denotes an argument minimum function to find the minimum distance $\left(\mathrm{d}_{\mathrm{ij}}\right)$ between the vehicle nodes and cluster mean. In this way, all the vehicle nodes are grouped into different clusters. After that, the mutual information between the clusters is computed for merging the clusters. The mutual information is used to compute the mutual dependence as given below.

$\mathrm{D}_{\mathrm{m}}=\mathrm{p}_{\mathrm{r}}\left(\mathrm{c}_{1}, \mathrm{c}_{2}\right) * \log _{2}\left(\left(\mathrm{p}_{\mathrm{r}}\left(\mathrm{c}_{1}, \mathrm{c}_{2}\right) / \operatorname{pr}\left(\mathrm{c}_{1}\right) \mathrm{p}_{\mathrm{r}}\left(\mathrm{c}_{2}\right)\right)\right)$

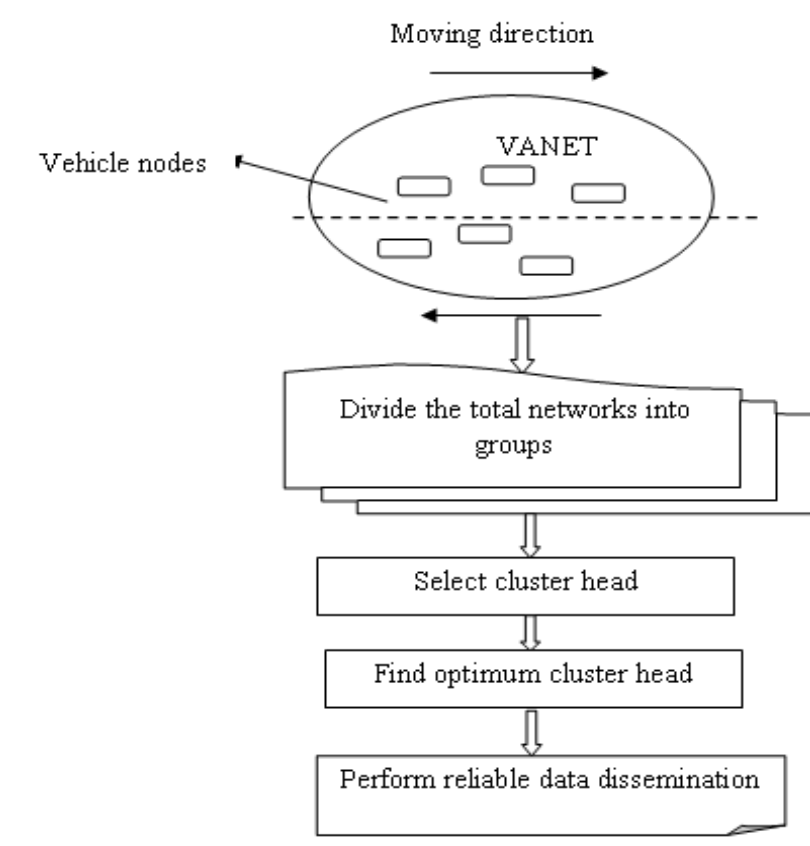

Fig. 2. Flow Process of Proposed MIBCMCSO Technique. 
Where, $D_{m}$ denotes a mutual dependence between the clusters $\left(\mathrm{c}_{1}, \mathrm{c}_{2}\right),\left(\mathrm{p}_{\mathrm{r}}\left(\mathrm{c}_{1}, \mathrm{c}_{2}\right)\right)$ denotes a joint probability distribution, and $\mathrm{p}_{\mathrm{r}}\left(\mathrm{c}_{1}\right)$ and $\mathrm{p}_{\mathrm{r}}\left(\mathrm{c}_{2}\right)$ represents a marginal probability of the clusters. The proposed clustering algorithm uses the gradient ascent function for finding the maximum mutual dependence between the clusters.

$\mathrm{G}(\mathrm{x})=\arg \max \mathrm{D}_{\mathrm{m}}$

Where, $\mathrm{G}(\mathrm{x})$ denotes a gradient ascent function, arg max represents an argument of the maximum function used to find the maximum mutual dependence $\mathrm{D}_{\mathrm{m}}$ between the clusters.

Finally, the merging process is carried out by combining the maximum dependence between the clusters. As a result, the number of clusters is obtained in output space. After that the cluster head is chosen through the minimum average distance among the other cluster members in the group. The cluster head acts as a data collector to route the information to their cluster members and to the roadside unit. The Mutual informated brown agglomerative clustering algorithm is described as follows:

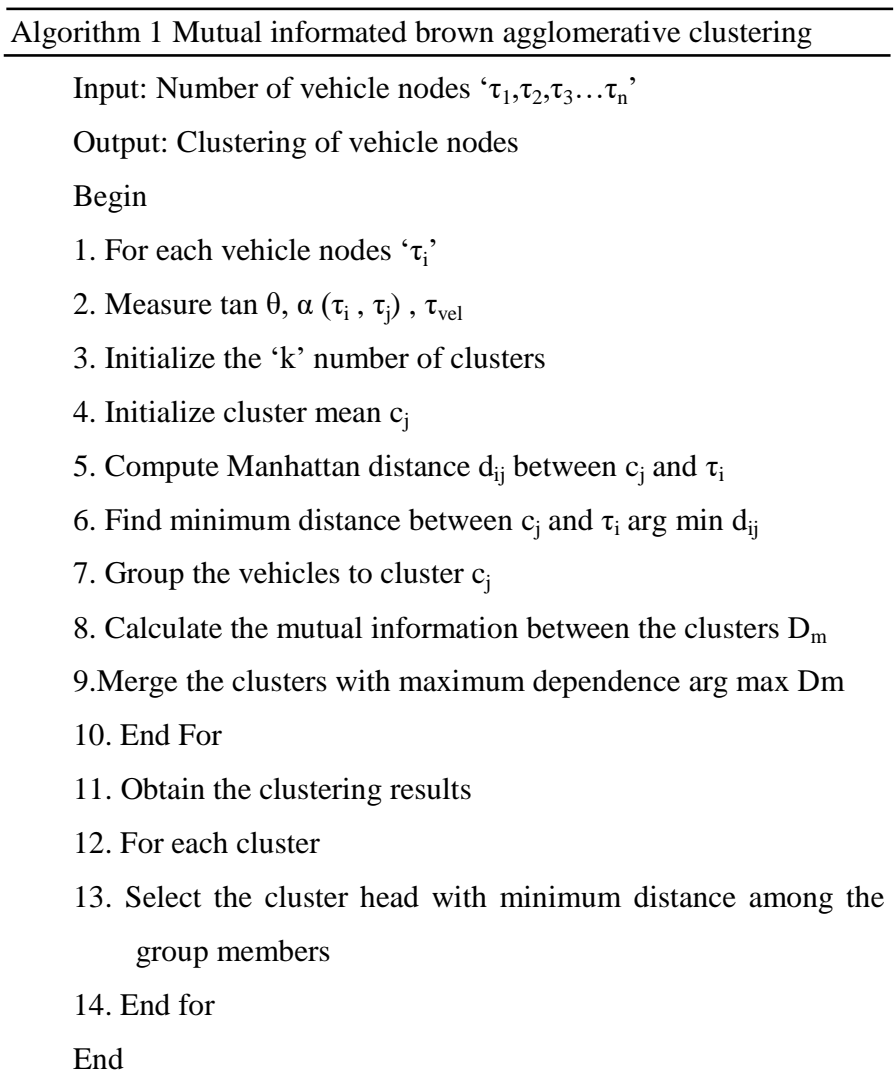

Algorithm 1 describes the step by step process of Mutual informated brown agglomerative clustering to group the vehicle nodes based on the node characteristics such as moving direction, distance, and velocity. The hierarchical clustering is applied for dividing the mobile nodes into different clusters. Then the cluster head is chosen for efficient data dissemination in VANET.

Multi-attribute cockroach swarm optimization was applied for detecting cluster head for data dissemination with minimum delay. The Multi-attribute cockroach swarm optimization algorithm is inspired by the basic biological behaviors of the cockroach looking for their food based on multiple objective functions namely, energy, bandwidth availability, and distance. Initialize the populations of cockroach i.e. cluster heads in the search space.

$\mathrm{C}_{\mathrm{s}}=\left\{\mathrm{Cr}_{1}, \mathrm{Cr}_{2}, \mathrm{Cr}_{3} \ldots \ldots \mathrm{Cr}_{\mathrm{n}}\right\}$

For each cluster head, the multiattribute function such as energy, bandwidth availability and distance is calculated to find the optimal one. The residual energy for each cluster head is mathematically estimated as given below:

$\mathrm{E}_{\mathrm{R}}=\left(\mathrm{E}_{\mathrm{t}}-\mathrm{E}_{\mathrm{c}}\right)$

Where, $E_{R}$ represents the residual energy, $E_{t}$ indicates total energy, $\mathrm{E}_{\mathrm{c}}$ denotes consumed energy. Then bandwidth accessibility of the cluster head is estimated as follows,

$\mathrm{B}_{\mathrm{A}}=\left(\mathrm{B}_{\mathrm{t}}-\mathrm{B}_{\mathrm{c}}\right)$

Where, $\mathrm{B}_{\mathrm{A}}$ denotes an available bandwidth between the cluster head, the total bandwidth is represented by $B_{t}$ amount of bandwidth utilization is represented by $B_{c}$.The distance between the two clusters head $\alpha\left(\tau_{\mathrm{i}}, \tau_{\mathrm{j})}\right.$ is calculated.

$\alpha\left(C_{i}, C_{j}\right)=\sqrt{\left(a_{2}-a_{1}\right)^{2}+\left(b_{2}-b_{1}\right)^{2}}$

Where $\left(a_{1}, b_{1}\right)$ and $\left(a_{2}, b_{2)}\right.$ denotes a location coordinates of two cluster heads in the two-dimensional space. Based on the above-said parameters, the fitness of each individual $\mathrm{Cr}_{\mathrm{i}}$ is computed.

$\mathrm{FF}=\left\{\left(\mathrm{E}_{\mathrm{R}}>\mathrm{E}_{\mathrm{T}}\right) \& \&\left(\mathrm{~B}_{\mathrm{A}}>\mathrm{B}_{\mathrm{T}}\right) \& \& \min \alpha\left(\mathrm{C}_{\mathrm{i}}, \mathrm{C}_{\mathrm{j}}\right)\right\}$

Where FF indicates the fitness of the individual in the search space, $E_{R}$ denotes residual energy, $E_{T}$ is the threshold for residual energy, $\mathrm{B}_{\mathrm{A}}$ indicates bandwidth availability, min $\alpha\left(\mathrm{C}_{\mathrm{i}}, \mathrm{C}_{\mathrm{j}}\right)$ denotes a minimum distance among two cluster heads. In addition, each iterations of proposed algorithm involves three behaviors for solving various optimization problems namely chase-swarming, dispersing, and ruthless behavior based on the fitness of the individual in search space.

1) Chase-swarming behaviors: In the chase-swarming behavior, the strongest cockroach among the population is considered as a local best solution $\left(\mathrm{x}_{1}\right)$ form the small swarms and move towards the global optimum $\left(\mathrm{x}_{\mathrm{g}}\right)$. Within this procedure, the local best solutions are taken randomly based on the best fitness. If the fitness of local best solutions $\left(\mathrm{x}_{1}\right)$ is greater than the $\left(x_{g}\right)\left(x_{g}\right)$ i.e. $F F\left(x_{l}\right)>F F\left(x_{g}\right)$, then the position of the local best solutions is updated to a global best solution,

$\mathrm{Cr}_{\mathrm{i}}=\mathrm{Cr}_{\mathrm{i}}+\delta * \mathrm{R} *\left(\mathrm{x}_{\mathrm{g}}-\mathrm{Cr}_{\mathrm{i}}\right)$

Where, $\mathrm{Cr}_{\mathrm{i}}$ denotes a current local best solutions are updated as to global best, $\delta$ denotes a step which is a fixed value, $\mathrm{R}$ indicates a random number within $[0,1], \mathrm{Cr}_{\mathrm{i}}$ is the local best individual position, and $\mathrm{x}_{\mathrm{g}}$ is a global best position. Otherwise, the cockroach $\mathrm{Cr}_{\mathrm{i}}$ goes to $\mathrm{x}_{1}$ (within its visibility) as given below,

$\mathrm{Cr}_{\mathrm{i}}=\mathrm{Cr}_{\mathrm{i}}+\delta * \mathrm{R} *\left(\mathrm{x}_{\mathrm{l}}-\mathrm{Cr}_{\mathrm{i}}\right)$ 
Where, $x 1$ denotes a local best solutions.

2) Dispersion: Another behavior is the dispersion of individuals which is carried out from time to time to maintain the diversity of cockroaches. Therefore, the behavior involves each cockroach performing a random step in the search space.

$\mathrm{Cr}_{\mathrm{i}}=\mathrm{Cr}_{\mathrm{i}}+\mathrm{R}(1, \mathrm{~d})$

Where, $\mathrm{R}(1, \mathrm{~d})$ denotes a d-dimensional random vector that the value is set within a certain range.

3) Ruthless behaviour: The final one is the Ruthless behavior which replaces the random individual into the current best individual (i.e. global best).

$\mathrm{Cr}_{\mathrm{k}}=\mathrm{x}_{\mathrm{g}}$

Where, $\mathrm{Cr}_{\mathrm{k}}$ is a random integer within $[0,1]$ and $\mathrm{x}_{\mathrm{g}}$ is the global best position. This procedure was frequent once highest amount of repetition is attained. In this way, global optimum cluster head was selected for data dissemination for reducing end to end delay. Algorithmic process of optimization-based cluster head selection as given below,

Algorithm 2 Multi-attribute cockroach swarm optimization

Input: Number of cluster heads $\mathrm{CH}_{1}, \mathrm{CH}_{2}, \mathrm{CH}_{3} \ldots \mathrm{CH}_{\mathrm{m}}$ (i.e. cockroaches)

Output: Select an optimal cluster head and perform data dissemination

Begin

1. Initialize the cockroaches populations $\mathrm{C}_{\mathrm{S}}=\left\{\mathrm{Cr}_{1}, \mathrm{Cr}_{2}, \mathrm{Cr}_{3} \ldots\right.$ $\left.\mathrm{Cr}_{\mathrm{n}}\right\}$ in search space

2. For each $\mathrm{Cr}_{\mathrm{i}}$

3. Measure the fitness FF

4. While $\left(\mathrm{t}<\mathrm{Max} \_\right.$iteration) do

5. $\operatorname{If}\left(\left(\mathrm{FF}\left(\mathrm{Cr}_{\mathrm{i}}\right)>\mathrm{FF}\left(\mathrm{x}_{\mathrm{g}}\right)\right)\right.$ then

6. $\mathrm{Cr}_{\mathrm{i}}=\mathrm{x}_{\mathrm{g}}$

7. Update the position $\mathrm{Cr}_{\mathrm{i}}=\mathrm{Cr}_{\mathrm{i}}+\delta * \mathrm{R} *\left(\mathrm{x}_{\mathrm{g}}-\mathrm{Cr}_{\mathrm{i}}\right)$

8. Else

9. $\mathrm{Cr}_{\mathrm{i}}=\mathrm{x}_{1}$

10. Update the position $\mathrm{Cr}_{\mathrm{i}}=\mathrm{Cr}_{\mathrm{i}}+\delta * \mathrm{R} *\left(\mathrm{x}_{1}-\mathrm{Cr}_{\mathrm{i}}\right)$

11. End if

12. for $i=1$ to $N$ do

13. $\mathrm{Cr}_{\mathrm{i}}=\mathrm{Cr}_{\mathrm{i}}+\mathrm{R}(1, \mathrm{~d})$

14. $k=$ random integer $([1, N])$

15. $\mathrm{Cr}_{\mathrm{k}}=\mathrm{x}_{\mathrm{g}}$

16. End for

17. $t=t+1$

18. Obtain the global best solution

19. Perform data dissemination via global best cluster head

End
Algorithm 2 shows the processes of Multi-attribute cockroach swarm optimization to select the optimal cluster head for efficient data dissemination with minimum delay. The number of individuals is selected among the population. Then, the fitness is calculated to choose the cluster head in the network based on different behaviors of cockroach. This process is iterated until a maximum number of iteration gets reached. Finally, the technique performs the data dissemination via the selected cluster head.

\section{Simulation Setup AND PARAMETER SetTingS}

The simulation of proposed MIBCMCSO technique and existing CEGRAOD [1] and Multi valued DPSO [2] are implemented using NS2.34 network simulator where the 500 vehicle nodes are deployed in a square area of A2 (1100 m* $1100 \mathrm{~m})$. The Random Waypoint model is used as node mobility in the simulation environment. The simulation time is set as $300 \mathrm{sec}$. The DSR protocol is used for cluster-based data dissemination in VANET. Table I describes the simulation parameters.

TABLE I. SIMULATION PARAMETERS AND VALUES

\begin{tabular}{|l|l|}
\hline Simulation Parameters & Values \\
\hline Network Simulator & NS2.34 \\
\hline Square Area & $1100 \mathrm{~m} * 1100 \mathrm{~m}$ \\
\hline Number of Vehicle Nodes & $50,100,150,200,250,300,350,400,450,500$ \\
\hline Number of Data Packets & $25,50,75,100,125,150,175,200,225,250$ \\
\hline Mobility Model & Random Waypoint Model \\
\hline Speed of Sensor Nodes & $0-20 \mathrm{~m} / \mathrm{s}$ \\
\hline Simulation Time & $300 \mathrm{sec}$ \\
\hline Protocol & DSR \\
\hline Number of Runs & 10 \\
\hline
\end{tabular}

Simulation of MIBCMCSO as well as existing technique were conducted by amount of vehicle nodes and data packets by different parameters are listed below.

\section{RESULT AND DISCUSSION}

The simulation result analysis of the proposed MIBCMCSO technique and existing CEGRAOD [1] and Multi valued DPSO [2] are discussed in this section with parameters such as packet delivery ratio, end to end delay and throughput. The different performance metrics results are discussed using tables and graphical representation.

\section{A. Impact of Packet Delivery Ratio}

Packet delivery ratio is known as reliability. It is referred by amount of data packets correctly received to entire number of data packets transferred via source node. It is mathematically calculated by,

$R=\left[\frac{\omega_{D R}}{n}\right] * 100$

Where number of data packet transferred is denoted as ' $n$ ' and amount of data packets successfully received is represented as ' $\omega_{\mathrm{DR}}$. It is calculated by percentage $(\%)$. 
TABLE II. PACKET DELIVERY RATIO

\begin{tabular}{|l|l|l|l|}
\hline \multirow{2}{*}{$\begin{array}{l}\text { Vehicle } \\
\text { Density }\end{array}$} & \multicolumn{3}{|l|}{ Packet Delivery Ratio $(\boldsymbol{\%})$} \\
\cline { 2 - 4 } & MIBCMCSO & CEGRAOD & $\begin{array}{l}\text { Multi Valued } \\
\text { DPSO }\end{array}$ \\
\hline $\mathbf{5 0}$ & 94 & 84 & 80 \\
\hline $\mathbf{1 0 0}$ & 95 & 86 & 82 \\
\hline $\mathbf{1 5 0}$ & 96 & 88 & 84 \\
\hline $\mathbf{2 0 0}$ & 95 & 89 & 85 \\
\hline $\mathbf{2 5 0}$ & 96 & 88 & 84 \\
\hline $\mathbf{3 0 0}$ & 97 & 90 & 85 \\
\hline $\mathbf{3 5 0}$ & 96 & 91 & 86 \\
\hline $\mathbf{4 0 0}$ & 95 & 90 & 85 \\
\hline $\mathbf{4 5 0}$ & 97 & 91 & 86 \\
\hline $\mathbf{5 0 0}$ & 96 & 89 & 85 \\
\hline
\end{tabular}

Table II illustrates packet delivery ratio of data transmission versus vehicle density varies from 50 to 500 . Reported results show packet delivery ratio is said to be improved using the MIBCMCSO technique as compared to other existing methods. Let us consider 50 vehicle densities for simulation, and 25 data packets are transferred via source node. Then destination node receives 24 data packets and the reliability of the MIBCMCSO technique is $96 \%$ whereas the reliably of the other two methods CEGRAOD [1] and Multi valued DPSO [2] are $84 \%$ and $80 \%$, respectively. Fig. 3 illustrates various results of packet delivery ratio.

Simulation results of packet delivery ratio have different vehicle density of three methods MIBCMCSO and existing CEGRAOD [1] and Multi valued DPSO [2] are explained from Fig. 3. Graphical outcome clearly describes packet delivery ratio of data transmission in VANET is said to be improved using the MIBCMCSO technique with two existing methods. This improvement is achieved by applying the cluster based optimization process. In the clustering process, the different groups of vehicle nodes are obtained for efficient data transmission. Then the optimum cluster head selection effectively delivers the data packets from source to destination. The multi attribute cockroach swarm optimization considers the bandwidth availability to transmit the data packets. The maximum bandwidth availability minimizes the packet drop and increases data delivery. Table III specifies the simulation results of packet delivery ratio.

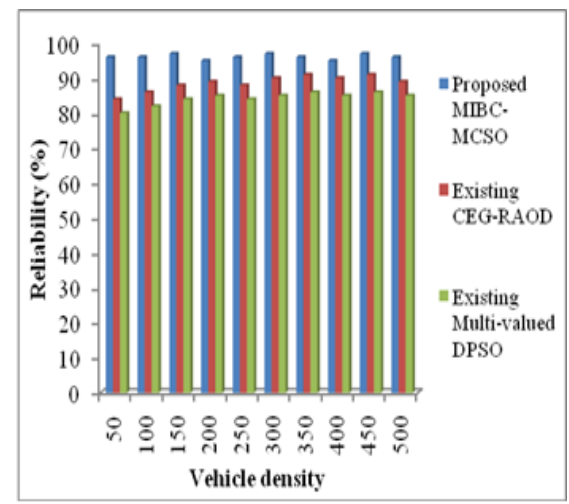

Fig. 3. Graphical Representation of Packet Delivery Ratio.
TABLE III. Simulation Results of Packet Delivery Ratio

\begin{tabular}{|l|l|l|}
\hline Parameters & CEGRAOD & Multi Valued DPSO \\
\hline MIBCMCSO & $14 \%$ & $9 \%$ \\
\hline
\end{tabular}

\section{B. Impact of End to End Delay}

It refers to time difference among packet arrival time as well as data packet transmitting time. Therefore overall delay is expressed by,

$D=\left\{t\left(D_{a}\right)-t\left(D_{s}\right)\right\}$

As shown in Table IV, end to end delay of MIBCMCSO technique as well as existing CEGRAOD [1] and Multi valued DPSO [2] is described. The reported result evidently confirms that MIBCMCSO technique of end to end delay is reduced with other existing methods. A graphical result of end to end delay is illustrated from Fig. 4.

Fig. 4 depicts graphical illustration of end to end delay by vehicle density. From illustration, end to end delay of data transmission is comparatively lesser by MIBCMCSO with existing results. This is because of the clustering-based data transmission. In order to perform data transmission, the whole network is divided into the number of groups using a mutual information brown agglomerative clustering technique. The data transmission is carried out only through the cluster head instead of sending all the nodes in the network. Therefore, the end to end delay from source to destination is minimized. Let us consider 50 nodes for simulation and 25 data packets are considered for calculating the delay. MIBCMCSO receives the data packets with $9 \mathrm{~ms}$ of delay and CEGRAOD [1] and Multi valued DPSO [2] receives the packets with $14 \mathrm{~ms}$ and $17 \mathrm{~ms}$ Similarly, the nine various results are carried out with respect to the number of nodes and different numbers of data packets. The simulation results of end to end delay are discussed in Table V.

\section{Impact of Throughput}

Throughput is referred by amount of data packets obtained on destination vehicle within specified time period. Therefore, throughput is expressed as follows,

$T=\left(\frac{\omega_{D S R}(\text { bits })}{\text { time }(\text { sec })}\right)$

TABLE IV. END TO END DELAY

\begin{tabular}{|l|l|l|l|}
\hline \multirow{2}{*}{$\begin{array}{l}\text { Vehicle } \\
\text { Density }\end{array}$} & \multicolumn{3}{|l|}{ End to End Delay } \\
\cline { 2 - 4 } & MIBCMCSO & CEGRAOD & Multi Valued DPSO \\
\hline $\mathbf{5 0}$ & 9 & 14 & 17 \\
\hline $\mathbf{1 0 0}$ & 11 & 16 & 19 \\
\hline $\mathbf{1 5 0}$ & 12 & 18 & 21 \\
\hline $\mathbf{2 0 0}$ & 15 & 22 & 25 \\
\hline $\mathbf{2 5 0}$ & 17 & 23 & 27 \\
\hline $\mathbf{3 0 0}$ & 19 & 25 & 29 \\
\hline $\mathbf{3 5 0}$ & 22 & 27 & 30 \\
\hline $\mathbf{4 0 0}$ & 23 & 30 & 33 \\
\hline $\mathbf{4 5 0}$ & 24 & 32 & 36 \\
\hline $\mathbf{5 0 0}$ & 27 & 34 & 38 \\
\hline
\end{tabular}




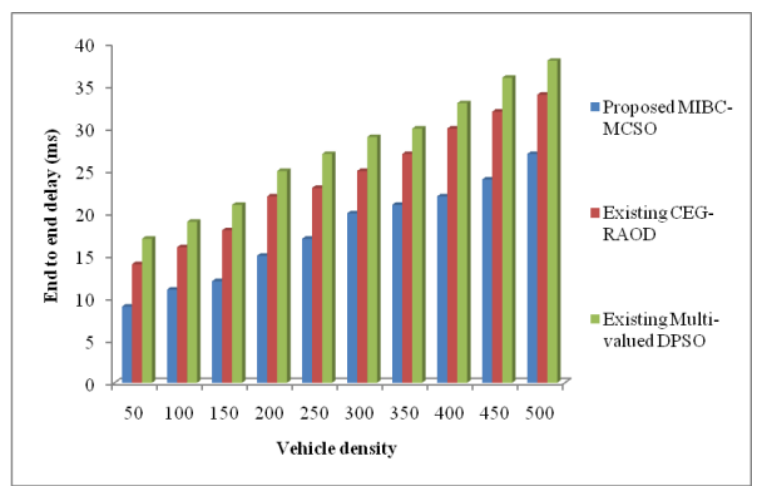

Fig. 4. Graphical Representation of End to End Delay.

TABLE V. Simulation Results OF END TO END DELAY

\begin{tabular}{|l|l|l|}
\hline Parameters & CEGRAOD & Multi Valued DPSO \\
\hline MIBCMCSO & $26 \%$ & $37 \%$ \\
\hline
\end{tabular}

Where, T represents the throughput, $\omega_{\mathrm{DSR}}$ (bits) $\omega_{D S R}$ (bits) indicates amount of data packets obtained on destination in terms of bits. It is measured by bits per second (bps).

The simulation result of throughput versus data packets is transferred via source node is illustrated in Table VI. For experimental conduction, sizes of data packets are taken in the range from 10KB-100KB. While varying the input sizes, different throughput were achieved. Outcomes are plotted in two-dimensional graphical representation.

Fig. 5 depicts a graphical representation of the throughput versus sizes of data packets. The various sizes of data packets are given as input to horizontal pivot and throughput were acquired on vertical pivot. Throughput is comparatively increased than the existing methods. This significant development of the MIBCMCSO technique is obtained by the optimal cluster head selection using multiattribute swarm optimization. The minimum distance, higher residual energy, and maximum bandwidth availability are said to improve the data dissemination from source to destination through the optimum cluster head. The throughput results are given in Table VII.

TABLE VI. THROUGHPUT

\begin{tabular}{|l|l|l|l|}
\hline \multirow{2}{*}{$\begin{array}{l}\text { Data Packet } \\
\text { Size(KB) }\end{array}$} & Throughput & \multicolumn{2}{|l|}{} \\
\cline { 2 - 4 } & MIBCMCSO & CEGRAOD & $\begin{array}{l}\text { Multi Valued } \\
\text { DPSO }\end{array}$ \\
\hline 10 & 156 & 100 & 95 \\
\hline 20 & 287 & 200 & 179 \\
\hline 30 & 395 & 315 & 280 \\
\hline 40 & 550 & 420 & 392 \\
\hline 50 & 610 & 500 & 452 \\
\hline 60 & 720 & 623 & 582 \\
\hline 70 & 820 & 710 & 653 \\
\hline 80 & 930 & 821 & 783 \\
\hline 90 & 1050 & 910 & 872 \\
\hline 100 & 1250 & 1052 & 986 \\
\hline
\end{tabular}

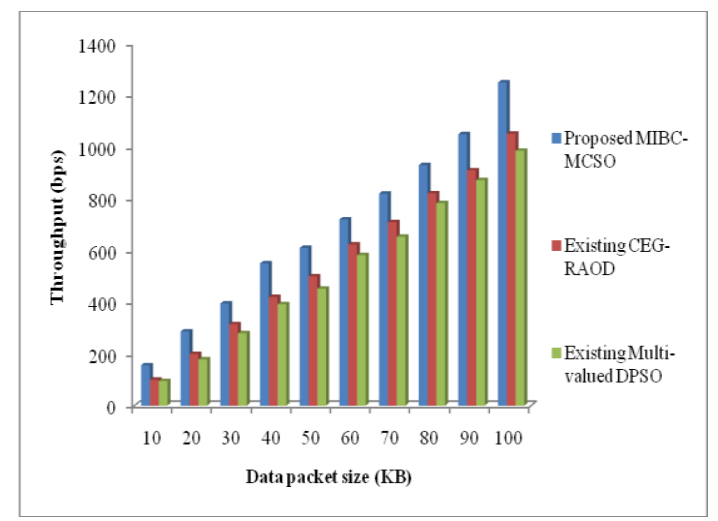

Fig. 5. Graphical Representation of Throughput.

TABLE VII. SIMULATION RESUlTS OF THROUGHPUT

\begin{tabular}{|l|l|l|}
\hline PARAMETERS & CEGRAOD & Multi Valued DPSO \\
\hline MIBCMCSO & $15 \%$ & $36 \%$ \\
\hline
\end{tabular}

The above discussion of various results of metrics evidently proves that the MIBCMCSO technique improves the higher packet delivery rate data dissemination with minimum delay as well as higher network throughput.

\section{CONCLUSION}

An efficient clustering-based optimization technique called MIBCMCSO is proposed for reliable data dissemination. MIBCMCSO technique employs mutual informative brown clustering to collect mobile nodes towards various groups for data dissemination with minimum delay. Followed by, optimum cluster head selection was carried out through mobility metrics such as distance, energy, and bandwidth. Simulation is conducted by various parameters namely packet delivery ratio, delay as well as throughput. MIBCMCSO increases packet delivery ratio of data dissemination into vehicle network and minimizes delay compared with conventional methods. In future, multi-criteria optimization problem in the cluster head selection such as link stability, and link expiration time are used.

\section{REFERENCES}

[1] Zahid Khan, Pingzhi Fan, Sangsha Fang and Fakhar Abbas," An Unsupervised Cluster-Based VANET-Oriented Evolving Graph (CVoEG) Model and Associated Reliable Routing Scheme",.IEEE Transactions on Intelligent Transportation Systems, pp. 1-16,2019.

[2] Manisha Chahal and Sandeep Harit," Optimal path for data dissemination in Vehicular Ad Hoc Networks using meta-heuristic", Computers \& Electrical Engineering, Elsevier,vol. 76,pp. 40-55,2019.

[3] Lei Liu, Chen Chen, Tie Qiu, Mengyuan Zhang, Siyu Li and Bin Zhou," A data dissemination scheme based on clustering and probabilistic broadcasting in VANETs",Vehicular Communications, Elsevier,vol. 13,pp. 78-88,2018.

[4] Shahid Latif, Saeed Mahfooz, Naveed Ahmad, Bilal Jan, Haleem Farman, Murad Khan, and Kijun Han," Industrial Internet of Things Based Efficient and Reliable Data Dissemination Solution for Vehicular Ad Hoc Networks", Wireless Communications and Mobile Computing, Hindawi,vol.2018,pp. 1-16,2018.

[5] Joahannes B. D. da Costa, Allan M. de Souza, Denis Rosário, Eduardo Cerqueira and Leandro A. Villas,"Efficient data dissemination protocol based on complex networks' metrics for urban vehicular networks",Journal of Internet Services and Applications,Springer. vol 10,pp. 1-13,2019. 
[6] Atif Ishtiaq, Sheeraz Ahmed, Muhammad Fahad Khan, Farhan Aadil, Muazzam Maqsood and Salabat Khan," Intelligent clustering using moth flame optimizer for vehicular ad hoc networks", International Journal of Distributed Sensor Networks, vol. 15,issue.1,pp. 1-13,2019.

[7] Shahid Latif, Saeed Mahfooz, Bilal Jan, Naveed Ahmad, Haleem Farman, Murad Khan, and Huma Javed,"Multicriteria Based Next Forwarder Selection for Data Dissemination in Vehicular Ad Hoc Networks Using Analytical Network Process",Mathematical Problems in Engineering,Hindawi,vol.2017,pp. 1-18,2017.

[8] Abdelali Touil and $\neg$ Fattehallah Ghadi," Efficient dissemination based on passive approach and dynamic clustering for VANET",Procedia Computer Science,Elsevier,vol.127,pp.369-378, 2018.

[9] Xiying Fan, Chuanhe Huang, Junyu Zhu and Bin Fu,"R-DRA: a replication-based distributed randomized algorithm for data dissemination in connected vehicular networks", Wireless Networks,Springer,.vol.25,issue.7,pp.3767-3782,2019.

[10] Min Xing, Jianping He, Lin Cai," Utility Maximization for Multimedia Data Dissemination in Large-Scale VANETs",IEEE Transactions on Mobile Computing,.vol. 16,issue. 4,pp. 1188-1198, 2017.

[11] Rene Oliveira, Carlos Montez, Azzedine Boukerche and Michelle S. Wangham," Reliable Data Dissemination Protocol for VANET Traffic Safety Applications",Ad Hoc Networks,Elsevier,vol.63,pp. 30-44,2017.

[12] Osama Rehman and Mohamed Ould-Khaoua," A hybrid relay node selection scheme for message dissemination in VANETs", Future Generation Computer Systems,Elsevier,vol.93,pp.1-17,2019.

[13] Sasirom Tiennoy and Chaiyachet Saivichit, "Using a Distributed Roadside Unit for the Data Dissemination Protocol in VANET With the Named Data Architecture", IEEE Access, Volume 6, 2018, Pages 32612- 32623.
[14] Yu-Yu Lin and Izhak Rubin, "Integrated Message Dissemination and Traffic Regulation for Autonomous VANETs", IEEE Transactions on Vehicular Technology, Volume 66, Issue 10, 2017, Pages 8644 - 8658.

[15] Chung-Ming Huang, Tzu-Hua Lin, Kuan-Cheng Tseng, "Data Dissemination of Application Service by Using Member-Centric Routing Protocol in a Platoon of Internet of Vehicle (IoV)", IEEE Access, Volume 7, 2019, Pages 127713 - 127727.

[16] A. A. Khan, M. Abolhasan, W. Ni, J. Lipman and A. Jamalipour, "A Hybrid-Fuzzy Logic Guided Genetic Algorithm (H-FLGA) Approach for Resource Optimization in 5G VANETs," in IEEE Transactions on Vehicular Technology, vol. 68, no. 7, pp. 6964-6974, July 2019.

[17] C. -M. Huang and C. -F. Lai, "The Delay-Constrained and NetworkSituation-Aware V2V2I VANET Data Offloading Based on the MultiAccess Edge Computing (MEC) Architecture," in IEEE Open Journal of Vehicular Technology, vol. 1, pp. 331-347, 2020.

[18] L. H. S. Lopes, R. A. F. Mini and F. Cunha, "A V2X Approach for Data Dissemination in Vehicular Ad Hoc Networks," 2019 IEEE Symposium on Computers and Communications (ISCC), 2019, pp. 1-6.

[19] Banoth Ravi, Jaisingh Thangaraj, "Stochastic traffic flow modeling for multi-hop cooperative data dissemination in VANETs", Physical Communication, Volume 46,2021.

[20] Huda Abualola, Hadi Otrok, Rabeb Mizouni, Shakti Singh,A V2V charging allocation protocol for electric vehicles in VANET,Vehicular Communications, 2021.

[21] L. Yang, L. Zhang, Z. He, J. Cao and W. Wu, "Efficient Hybrid Data Dissemination for Edge-Assisted Automated Driving," in IEEE Internet of Things Journal, vol. 7, no. 1, pp. 148-159, Jan. 2020. 